\title{
The General Self-Efficacy Scale: Multicultural Validation Studies
}

\author{
ALEKSANDRA LUSZCZYNSKA \\ School of Life Sciences, Department of Psychology \\ University of Sussex, UK \\ and \\ Warsaw School of Social Psychology, Poland \\ URTE SCHOLZ \\ RALF SCHWARZER \\ Department of Health Psychology \\ Freie Universität Berlin, Germany
}

\begin{abstract}
General self-efficacy is the belief in one's competence to cope with a broad range of stressful or challenging demands, whereas specific self-efficacy is constrained to a particular task at hand. Relations between general self-efficacy and social cognitive variables (intention, implementation intentions, outcome expectancies, and self-regulation), behavior-specific self-efficacy, health behaviors, well-being, and coping strategies were examined among 1,933 respondents in 3 countries: Germany $(n=$ 633 ), Poland $(n=359)$, and South Korea $(n=941)$. Participants were between 16 and 86 years old, and some were dealing with stressful situations such as recovery from myocardial events or tumor surgery. Perceived self-efficacy was measured by means of the General Self-Efficacy Scale (R. Schwarzer \& M. Jerusalem, 1995). Meta-analysis was used to determine population effect sizes for four sets of variables. Across countries and samples, there is consistent evidence for associations between perceived self-efficacy and the variables under study confirming the validity of the psychometric scale. General self-efficacy appears to be a universal construct that yields meaningful relations with other psychological constructs.
\end{abstract}

Key words: coping, cross-cultural research, health behavior, implementation intention, outcome expectancies, self-efficacy

ACCORDING TO SOCIAL COGNITIVE THEORY (SCT; Bandura, 1997), human motivation and actions are regulated extensively by forethought. The prime factor for influencing behavior is perceived self-efficacy, that is, people's beliefs in their capabilities to perform a specific action required to attain a desired outcome. Self-efficacy is of a prospective and operative nature (e.g., "I am certain that I can exercise regularly, even if my exercise partner returns 
to a sedentary lifestyle"). Thus, perceived self-efficacy can be characterized as being competence-based, prospective, and action-related (Bandura).

Self-efficacy is usually understood as being either task specific or domain specific. However, some researchers have also conceptualized a generalized sense of self-efficacy that refers to a broad and stable sense of personal competence to deal effectively with a variety of stressful situations (Schwarzer \& Jerusalem, 1995; Sherer et al., 1982). General self-efficacy (GSE) reflects a generalization across various domains of functioning in which people judge how efficacious they are. For the majority of applications, perceived self-efficacy should be conceptualized in a situation-specific manner (Bandura, 1997). GSE, however, may explain a broader range of human behaviors and coping outcomes when the context is less specific. It might be useful when focusing on multiple behaviors simultaneously (Luszczynska, Gibbons, Piko, \& Tekozel, 2004) or when studying the well-being or behavior of patients who have to adjust their lives to multiple demands owing to illness (Bonetti et al., 2001).

In the present study, we explored the relations between GSE and a variety of other psychological constructs. We describe why and how certain variables should be related to GSE. According to SCT, people are considered to be selforganizing, self-reflective, and self-regulative in that they make judgments about themselves on the basis of their own activity. Moreover, self-efficacy beliefs influence other cognitions, affect, and behaviors and may also help to deal with stressful circumstances (Bandura, 1997).

GSE is a universal construct, which means that it characterizes a basic belief that is inherent in all individuals. A cross-cultural commonality of beliefs about efficacy to produce effects by personal action might be expected (Bandura, 2002). Therefore, one might assume that associations between self-efficacy and related constructs would be similar across cultures and samples.

\section{Relations Between General Self-Efficacy and Social-Cognitive Constructs}

SCT implies that self-efficacy is the crucial and proximal predictor of behavior. Its influence on behavior is also indirect. Self-efficacy affects goals and outcome expectancies that are also predictors of behavior (Bandura, 1997, 2002).

Self-efficacy beliefs affect behaviors indirectly through their impact on goal intentions. The structure "I intend to" or "I aim to" reflects proximal goals (or intentions). Self-efficacy, among other factors, influences which

The authors thank Britta Renner, Sunkyo Kwon, Youlia Spivak, Nihal E. Mohamed, Adriana Zagorska, Jakub Szapiro, and Beata Kuzimska for their collaboration on this project. Aleksandra Luszczynska was an Alexander von Humboldt Fellow and Hertie Fellow.

Address correspondence to Aleksandra Luszczynska, School of Life Sciences, Department of Psychology, University of Sussex, Pevensey Building 1, Falmer, Brighton BN19QH, UK; A.Luszczynska@sussex.ac.uk (e-mail). 
challenges people decide to meet and how high they set their goals. Persons with high self-efficacy in a specific domain select more challenging and ambitious goals. High self-efficacy not only improves goal setting, but it also leads to more persistence in pursuing the goal. Therefore, self-efficacious individuals have stronger intentions.

According to Gollwitzer (1999), implementation intentions (or action plans) refer to the translation of goals or intentions into specific actions (How should I behave?) and into the situational circumstances of performance (Where and when should I perform the action?). Self-efficacious individuals focus on their future and develop possible success scenarios of their actions. Therefore, they are expected to be more committed to planning.

Outcome expectancies pertain to the perception of possible consequences of one's action (Bandura, 1997). Outcome expectancies refer to positive or negative consequences of specific actions (for example, engaging in regular physical activity). Negative outcome expectations, such as the prospect of discomfort or the necessity to abstain from other favorite activities, represent the anticipation of adversity after a behavior change takes place. Positive outcome expectancies refer to benefits of a behavior change (e.g., weight reduction or satisfaction with one's behavior). According to SCT, strong self-efficacy is related to perceiving more positive outcomes of future actions and fewer negative outcomes.

Self-efficacy describes individuals' beliefs in their capabilities to exercise control over challenging demands and over their own functioning. Self-regulation refers to any effort a person makes to alter his or her own responses, overriding impulses and substituting them with another response that leads toward a selected goal (cf. Luszczynska, Diehl, Gutiérrez-Doña, Kuusinen, \& Schwarzer, 2004). Thus, persons with high self-regulation are expected to be highly self-efficacious.

In the present study, we investigated relations between GSE and SCT constructs. Previous studies on self-efficacy focused on relations between SCT constructs and domain-specific self-efficacy (for a review, see Contento, Randell, \& Basch, 2002). GSE beliefs might be related to general self-regulatory cognitions (such as self-regulation of attention) as well as to cognitions that are specific for certain domains (such as outcome expectancies regarding physical activity). One might expect that GSE would be related to specific types of self-efficacy (Cervone, 1997).

\section{Self-Efficacy and Its Relations to Well-Being, Health Behaviors, and Coping}

Self-efficacy has a regulatory function in different health domains, such as adherence to medical recommendations (e.g., adoption of a physically active lifestyle), positive and negative affect, dealing with pain, and coping with stress.

According to SCT, the adoption of health-promoting behavior depends on beliefs of being able to perform a specific behavior appropriately (Bandura, 
1997). Individuals with stronger self-efficacy are more likely to engage in healthy behaviors, to maintain them, and to recover after setbacks.

Optimistic self-beliefs about one's own competence create positive affective states instead of negative ones, such as anxiety. Self-efficacious individuals may experience a low level of negative emotions in a threatening situation and, as a result, may feel capable of mastering the situation (Bandura, 1997). Individuals who are burdened with self-doubts suffer distress and negative emotions, such as anxiety and depression (Bandura). In addition, negative emotions may generate cognitive confusion, leading to worse solutions to the problems.

Patients' beliefs about their ability to cope with and tolerate pain are predictors of pain behaviors, pain intensity, and pain tolerance (Bandura, 1997). Those who are self-efficacious engage more easily in different activities in spite of pain, thus reducing pain intensity and disability. Individuals with high self-efficacy might therefore cope more actively with pain, which leads to a reduction of perceived intensity of pain.

Perceived self-efficacy represents the confidence that one can use the skills necessary to resist temptation, cope with stress, and mobilize resources required to meet the situational demands (Bandura, 1997). Once an action has been taken, people with high self-efficacy invest more effort and persist longer than those who are low in self-efficacy. Therefore, one might expect that the former would select active coping strategies and use fewer strategies that are passive or focused on negative emotions than the latter. People with strong self-efficacy recognize that they are able to overcome the obstacles and focus on opportunities. Self-efficacy leads to effective problem solving (Bandura).

\section{The Aims of the Study}

In the present study, we examined the hypothesized relations between GSE and the variables that were described earlier. According to SCT (Bandura, 1997), GSE should be related to certain social-cognitive constructs, such as goal intentions, implementation intentions, and outcome expectancies. We hypothesized that GSE would be related to domain-specific optimistic self-beliefs (such as physical activity self-efficacy, nutrition self-efficacy, or smoking self-efficacy). We also hypothesized that individuals with high GSE should have lower levels of negative affect and a higher quality of life, perceive lower intensity of pain, and perform healthy behaviors more frequently. The final hypothesis refers to relations between GSE and coping with stressful situations: We expected that self-efficacy would be related to more frequent use of active, problem-focused coping strategies and less frequent use of passive coping strategies.

We expected that the countries included in the study might vary in terms of culture in ways that reflect their differences in economic development, religion, and current and past social and political situations. The study included an economically developed country (Germany), a post-communist developing country (Poland), and 
a fast-developing country in Asia (South Korea). Samples included individuals dealing with different stressful situations such as sport competition or hospitalization, as well as individuals facing adaptation to a new life situation (e.g., patients after cancer surgery or patients discharged from rehabilitation clinics).

\section{Method}

\section{Participants and Procedure}

The GSE scale was completed by 1,933 respondents in three countries: Germany $(n=633)$, Poland $(n=359)$, and South Korea $(n=941)$. Several samples in both Germany and Poland were collected and combined. Respondents did not receive any compensation for participating in the study. The questionnaires were self-administered under the supervision of research team members, who were responsible for ensuring confidentiality and who answered participants' questions.

The first German sample consisted of 395 patients with coronary heart disease. Data were collected 2 months after their discharge from three rehabilitation centers in Berlin, Germany. Participants were 31 to 82 years old $(M=58.6$ years, $S D=$ $10.01)$. The majority $(n=316 ; 80 \%)$ were men. Most participants were married or living with a partner $(n=304 ; 77 \%), 51(12.9 \%)$ were single or widowed, $34(8.6 \%)$ were divorced, and $6(1.5 \%)$ did not indicate their family status. The majority $(n=$ $329 ; 83.3 \%$ ) had children. Of all participants, $29.4 \%$ reported 9 years, $21 \% 10$ years, $25.3 \% 12$ years, and $20.3 \% 13$ years of education; $4 \%$ did not indicate their education level. Approximately half of the respondents (48.1\%) were employed.

The second German sample consisted of 238 patients with cancer. Data were collected 1 month after surgery in four cancer treatment centers in Berlin. Most of the patients were treated for gastrointestinal cancer $(29 \%$ had cancer of the colon, $27 \%$ of the rectum, $11 \%$ of liver and gall bladder, and $12 \%$ of the pancreas). Participants were 29 to 86 years old $(M=62$ years, $S D=11.4)$. The majority $(n=136 ; 57 \%)$ were men. Most of the participants were married or living with a partner $(n=179 ; 75 \%)$, and $200(84 \%)$ reported having children.

The South Korean sample consisted of 941 persons who completed the questionnaires in Seoul in 2004. Participants were 17 to 91 years old ( $M=30.87$ years, $S D=15.87)$. The majority $(n=540 ; 57.4 \%)$ were women. Most of the participants were single ( $n=568 ; 60.4 \%$ ), 1.3\% (9 persons) were divorced, $297(31.6 \%)$ were married or remarried, and $62(6.6 \%)$ were widowed. Most reported education beyond high school (46\%), 44.6\% had finished high school, and $9.4 \%$ had finished school beyond the high school level. Approximately half of the participants were currently employed $(45.9 \%)$.

The first Polish sample consisted of 225 students at three universities in Warsaw, Poland. The students, of whom 110 (49\%) were men, were 19 to 26 years old $(M=21$ years, $S D=1.51)$. All participants had at least 13 years of education. Data were collected during classes on a voluntary basis. 
The second Polish sample consisted of 54 professional swimmers $(n=36$; $64 \%$ men), aged 15 to $26(M=17.6$ years, $S D=1.64)$, who participated in regular training in four centers for at least 6 years. Data were collected one day before regional championships. All participants were approached individually.

The third Polish sample consisted of 80 patients with gastrointestinal diseases ( $50 \%$ men) hospitalized at internal disease units of four hospitals in Warsaw. They were 35 to 65 years old $(M=49.44$ years, $S D=9.97) ; 44 \%$ had 12 years of education, and $29 \%$ between 8 and 11 years. Patients were admitted to hospitals with pain complaints due to various chronic or acute diseases (e.g., appendicitis, cholilithiasis). They were interviewed individually and took part in the study on a voluntary basis. Table 1 displays the number and the mean age of participants, by nation and gender.

\section{Measures}

The GSE scale includes 10 items. It was originally developed in Germany over the course of two decades, and in the meantime has been adapted to 28 languages (Schwarzer \& Jerusalem, 1995). A typical item is, "Thanks to my resourcefulness, I can handle unforeseen situations." Possible responses are not at all true (1), hardly true (2), moderately true (3), and exactly true (4), yielding a total score between 10 and 40 . Bilingual native speakers adapted the self-efficacy items to foreign languages based on the German and English versions of the GSE scale (Scholz, Gutierrez-Doña, Sud, \& Schwarzer, 2002). The adaptations followed the "group consensus model," including back translations and group discussions (Brislin, 1970).

High reliability, stability, and construct validity of the GSE scale were confirmed in earlier studies (Leganger, Kraft, \& Røysamb, 2000; Schwarzer, Mueller, \& Greenglass, 1999). The scale was found to be configurally equivalent across 28 nations, and it forms only one global dimension (Leganger et al.; Scholz et al., 2002).

In the present study, the following Cronbach alphas were obtained for the GSE scale: .94 (patients with cardiovascular diseases, Germany), .89 (patients with cancer, Germany), .90 (students, Poland), .87 (patients with gastrointestinal diseases, Poland), .87 (swimmers, Poland), .86 (participants from South Korea). Means and standard deviations of the scale are displayed in Table 1.

A set of measures to assess behavior-specific self-efficacy, social-cognitive constructs, well-being, health behaviors, and coping strategies was chosen for each of the samples. Only measures with satisfactory psychometric properties were included. Names of scales, item examples, numbers of items, response format, and Cronbach alphas for all samples are displayed in Table 2.

\section{Statistical Analyses}

We computed Pearson correlations and treated missing values by pairwise deletion. For the entire study, we set the Type I error level to .05. However, sta- 


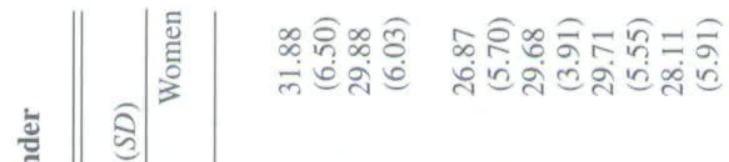

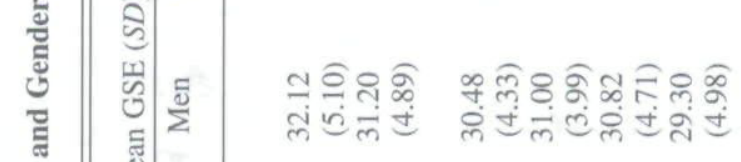

$$
\begin{aligned}
& \text { ह } \sum^{\mathrm{E}}
\end{aligned}
$$

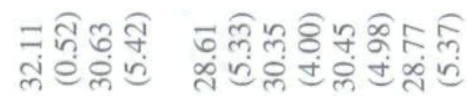

$$
\begin{aligned}
& \text { s }
\end{aligned}
$$

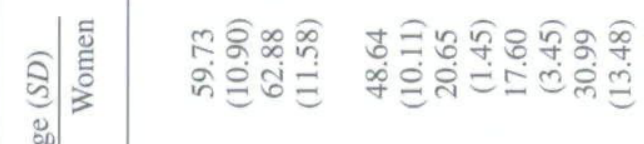

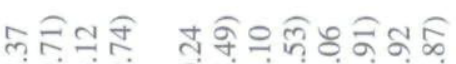

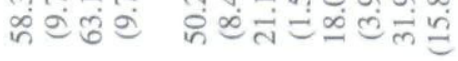

$$
\begin{aligned}
& \text { 胥 }
\end{aligned}
$$

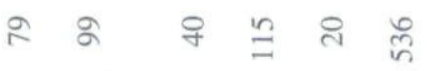

$$
\begin{aligned}
& \text { हँ }
\end{aligned}
$$

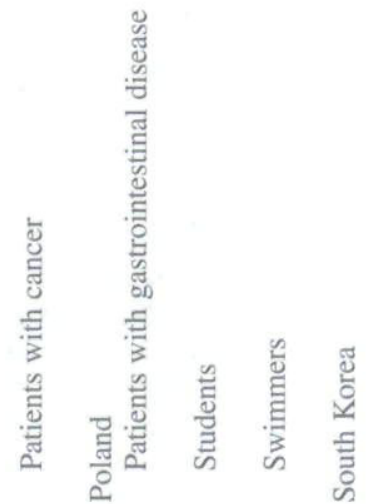




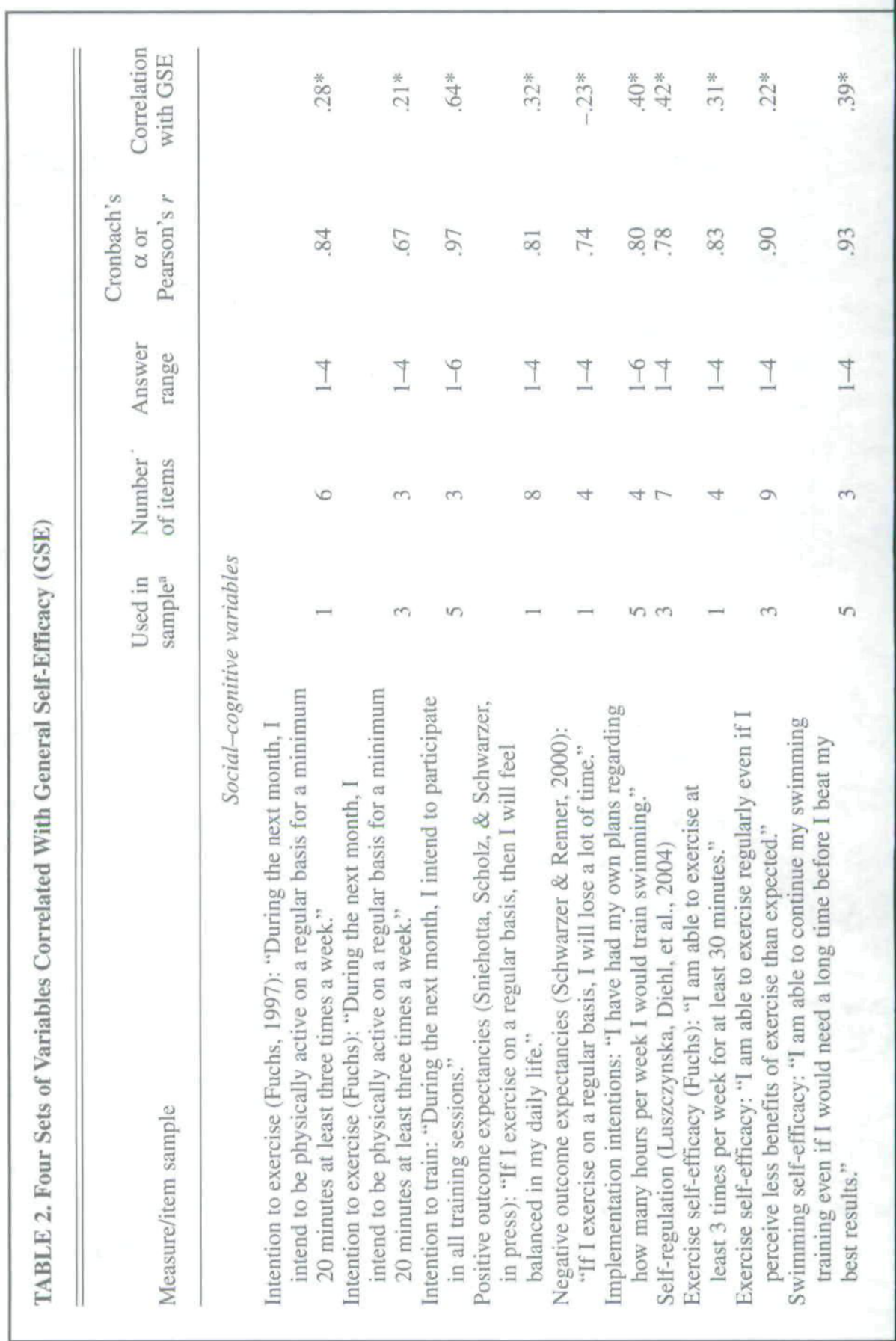




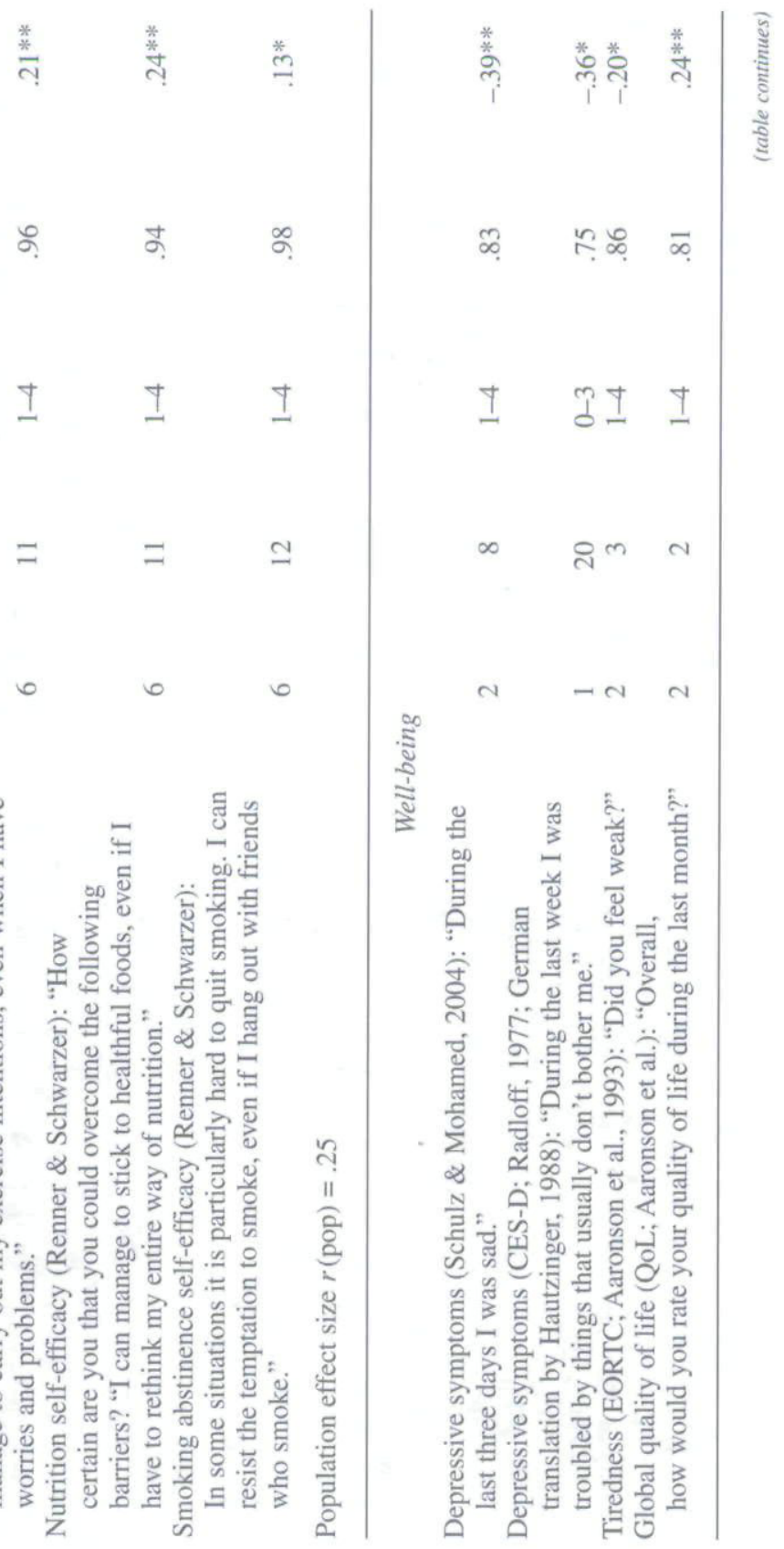




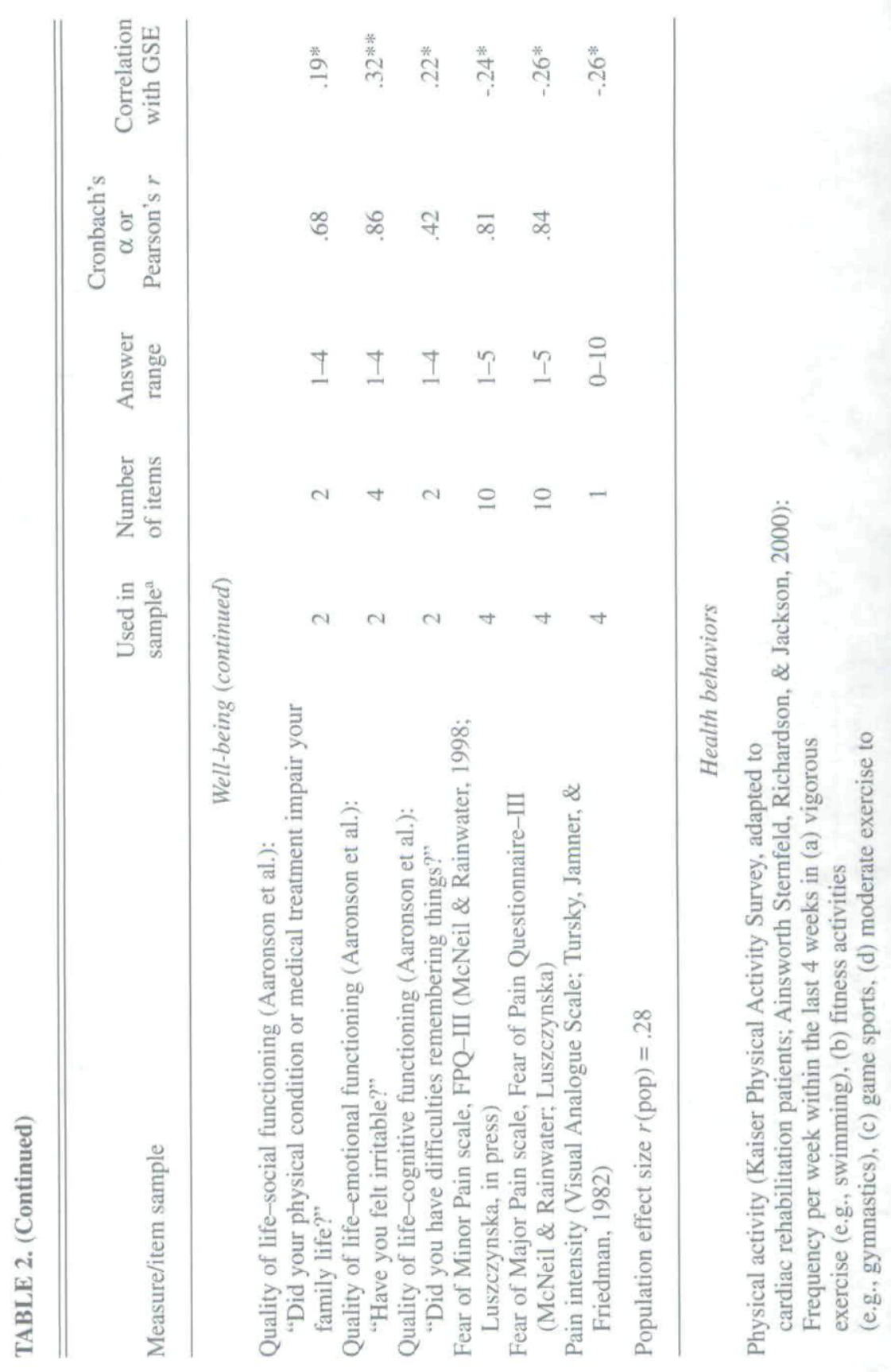




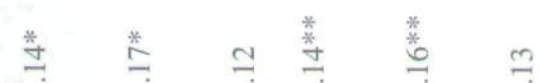

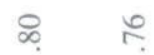

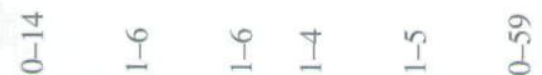

刃.

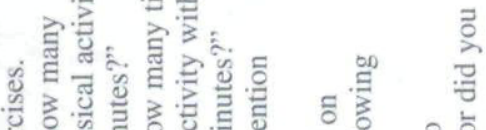

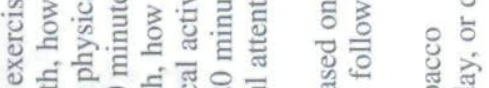

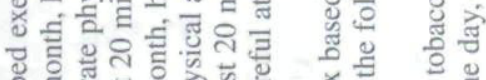

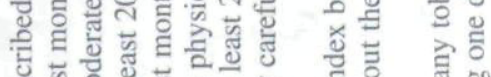

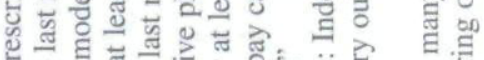

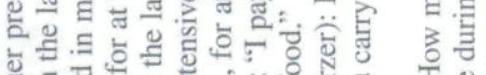

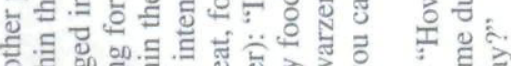

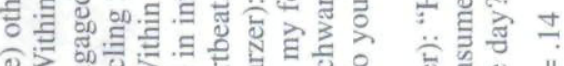

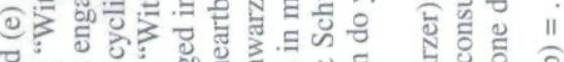
ํ..을

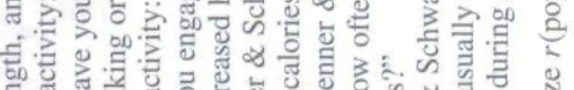

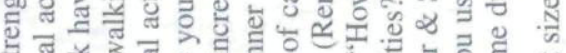

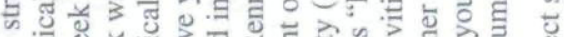

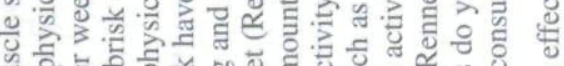

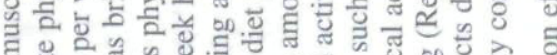

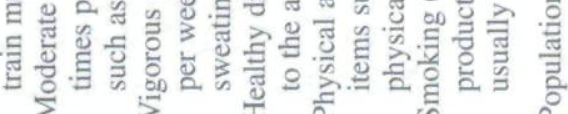

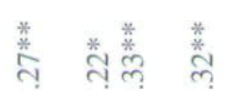

离古吊

$\pm \pm \pm \pm$

N N N

$\mathrm{NN} N$

ส ญ एँ

尊 ह

당 는

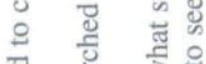

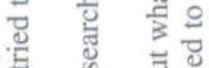

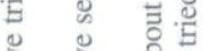

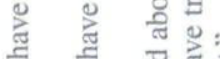

Е

$\because \quad$ 药 吾

ล

-

过

焉

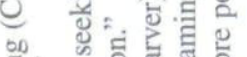

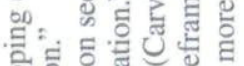

ㅎํㅇ.을 클

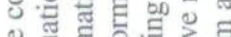

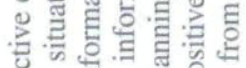




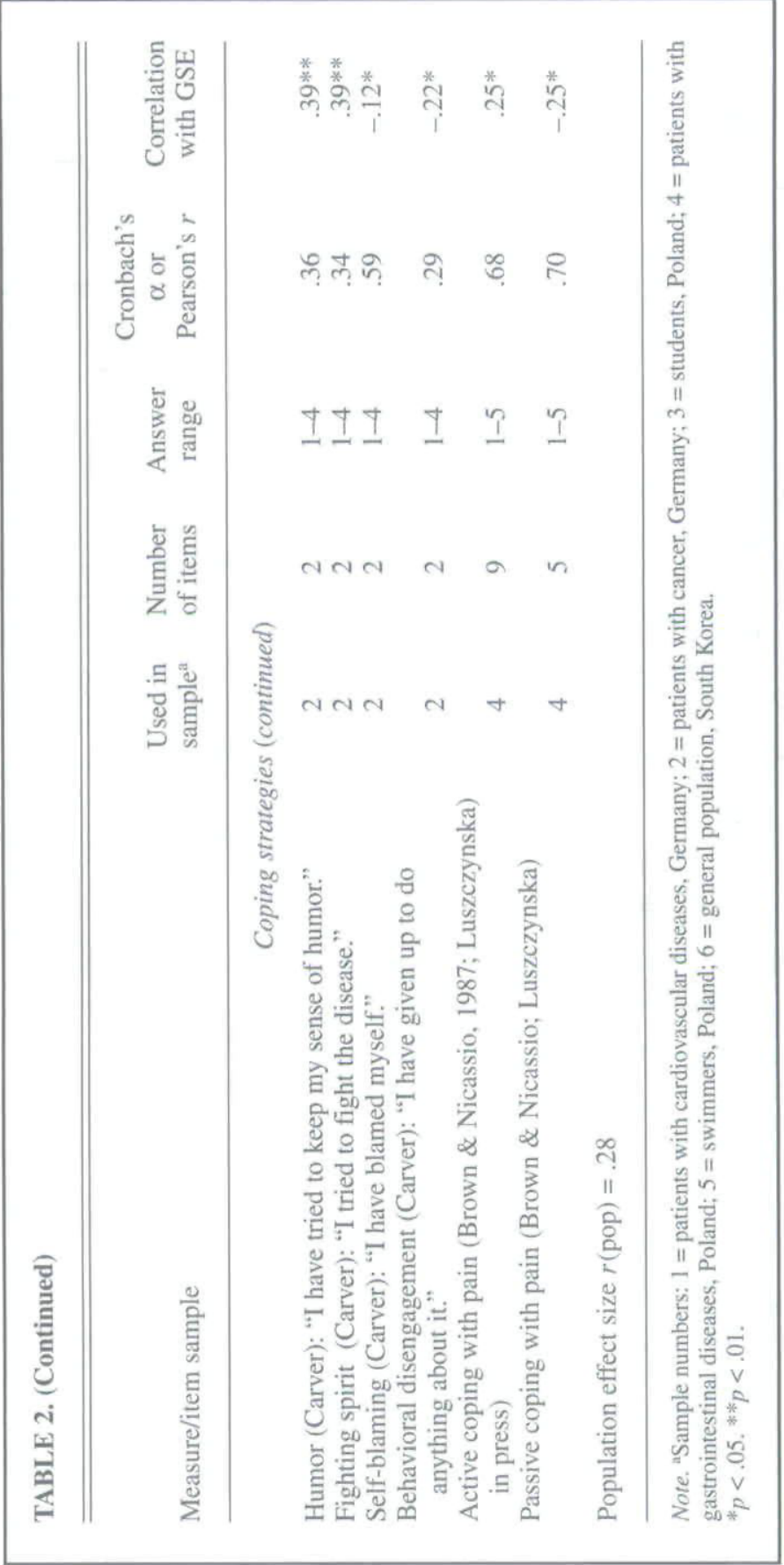


tistical significance is not the issue here, because it depends on sample size and computed within-samples multiple correlations. Instead, in this study, the focus was on effect size. For this purpose, we computed four meta-analyses, using the Schmidt-Hunter method for the synthesis of effect sizes ( $r \mathrm{~s}$; Hunter, Schmidt, \& Jackson, 1982), performed with meta-analysis software (Version 5.3; Schwarzer, 1991). In Table 2, we report population effect sizes (weighted by sample size) along with the corresponding effect size $(d)$ and the homogeneity of data. Metaanalytic findings are judged as homogeneous when two out of the following three criteria are met: (a) the residual $S D$ is smaller than one fourth of population effect size, (b) the percentage of residual variance is less than $25 \%$, and (c) a chi-square test of homogeneity is not significant.

\section{Results}

We computed relations between social-cognitive variables, such as goal intention, implementation intention, outcome expectancies, and self-regulation of attention and GSE. Among patients with cardiovascular diseases and among students, higher GSE was related to a stronger intention to exercise. In swimmers, GSE was associated with a stronger intention to continue training. Athletes with high GSE were more engaged in developing implementation intentions regarding the frequency and length of swimming training. Patients with cardiovascular diseases who had strong general optimistic self-beliefs expected more positive and fewer negative outcomes of engaging in regular physical activity. Students with high GSE reported stronger self-regulation of attention. The correlation coefficients are displayed in Table 2.

Across the samples, GSE beliefs were positively related to behavior-specific self-efficacy beliefs. Individuals with cardiovascular diseases who had higher GSE reported stronger optimistic self-beliefs about their ability to exercise in spite of barriers. Similar relations between these two variables were found among students. Swimmers with high GSE had stronger beliefs about their ability to continue their training. In the South Korean sample, GSE beliefs were related to nutrition self-efficacy, exercise self-efficacy, and smoking abstinence self-efficacy. The correlation coefficients are displayed in Table 2.

\section{General Self-Efficacy, Well-Being, and Health Behaviors}

As hypothesized, we found negative correlations between GSE and negative affect. Strong general efficacy beliefs were related to lower levels of depression in patients with cardiovascular diseases and to lower levels of anxiety in individuals with gastrointestinal diseases. The latter also reported lower levels of pain intensity. In patients with cancer, efficacious individuals were less depressed and less tired. They reported better health and better quality of life in the areas of emotional, social, and cognitive functioning. The correlation coefficients are displayed in Table 2. 
We also analyzed relations between health behaviors and GSE. Students with high GSE performed moderate physical activity more frequently than their counterparts with low GSE. However, the relations with vigorous physical activity were nonsignificant. In cardiovascular disease cases, individuals with high GSE were also physically more active. South Koreans with high GSE performed physical activity and ate a healthy diet more regularly than those with low GSE. The relation between number of cigarettes smoked daily and GSE was nonsignificant for the South Korean subsample of smokers. The correlation coefficients are displayed in Table 2.

\section{General Self-Efficacy and Coping}

According to the structural features described earlier, we expected that higher GSE should be related to more active coping with various difficult situations. Among cases of gastrointestinal disease, GSE was related to less frequent use of passive coping and more frequent use of active coping with pain. Among patients with cancer, GSE was related to the more frequent use of active coping, planning, positive reframing, humor, and fighting spirit, and to a more frequent seeking of information. Patients with cancer who had lower GSE more frequently used coping strategies such as self-blame or behavioral disengagement. The correlation coefficients for GSE and coping strategies are also displayed in Table 2.

\section{Meta-Analytic Results}

We performed the first meta-analysis on the initial 13 effect sizes (absolute values) listed in Table 2 that dealt with social-cognitive variables. A population effect size of $r(\mathrm{pop})=.25$ emerged, which is equivalent to $6 \%$ of shared variance between GSE and the social-cognitive variables. It is also equivalent to $d=.50$, which means that highly self-efficacious individuals do half a standard deviation better in terms of social-cognitive variables than their low self-efficacious counterparts. However, this first set of results did not attain homogeneity, and, therefore, it evaded further interpretation.

For the second meta-analysis, we aggregated 10 correlations between GSE and well-being and found a population effect size of $r$ (pop $)=.28$, equivalent to $d=.58$. This result was homogeneous. The coefficient of .28 , therefore, reflects very well the relationship between GSE and the selected emotional variables.

The third meta-analysis integrated the six coefficients representing the health behavior-GSE relationship. Here, the population effect size was $r(\mathrm{pop})=$ .14 , equivalent to $d=.29$, which was perfectly homogeneous, reflecting the low, but consistent, association between these sets of variables.

We performed the last meta-analysis on the 10 correlations between coping and GSE. Again, a homogeneous, that is, trustworthy, result with $r$ (pop) $=.28$, equivalent to $d=.58$, emerged. 


\section{Discussion}

Across countries, as hypothesized, GSE was related to the selected constructs. The correlations between GSE and social-cognitive variables, such as goal intentions, implementation intentions, outcome expectancies, self-regulation, and domain-specific self-efficacy remained significant and in the low range. Therefore, the amount of variance shared between these variables was low in most cases.

These results are in line with earlier studies on relations between self-efficacy and other social-cognitive constructs. Previous studies, however, have focused on relations between SCT constructs and domain-specific self-efficacy. Usually, relations for domain-specific self-efficacy, intentions, implementation intentions, and outcome expectancies were of moderate range (Luszczynska \& Schwarzer, 2003; Petosa, Suminski, \& Hortz, 2003), whereas in this study, the majority of correlation coefficients between those variables was lower, probably because of the generality of the self-efficacy construct used in the present study. Stronger relations were found for GSE and other general constructs, such as selfregulation, than for GSE and behavior-specific constructs, such as outcome expectancies.

General optimistic self-beliefs are not connected with a specific behavior, and therefore they may be a robust predictor of a variety of health behaviors. Previous researchers did not use GSE jointly with domain-specific cognitions, although researchers suggested the necessity to investigate such relations as well (cf. Cervone, 1997). Only low relations between GSE and domain-specific selfefficacy were found in the present study. General and specific optimistic selfbeliefs, beyond some commonalities, form two distinct social-cognitive constructs. The results of the present study thus provide evidence for discriminant validity for GSE, because it is weakly associated with behavior-specific constructs.

Results of previous studies using GSE beliefs as predictors of behaviors, such as physical activity (cf. Luszczynska, Gibbons, et al., 2004), and those that used domain-specific self-beliefs, that is, exercise self-efficacy (Rodgers, Hall, Blanchard, McAuley, \& Munroe, 2002), yielded clearly different prediction results. Therefore, one should not imply from studies on domain-specific selfefficacy that similar results would be obtained for GSE.

The second group of variables consisted of measures of affect, pain, quality of life, and health behaviors. As mentioned previously, according to SCT, one expects high self-efficacy to be related to low negative affect, high positive affect, more life satisfaction, and lower pain. All the relations found in the present study were significant but low. The associations between GSE and positive and negative affect were similar to previous findings. Associations between GSE, anxiety, and depression were found among students (Ashby \& Kottman, 2000; Leganger et al., 2000) and among people who were sick (Rimm \& Jerusalem, 1999). GSE 
predicted quality of life in patients with a spinal cord injury (Hampton, 2000). Pain-specific self-efficacy was negatively related to pain intensity (Brekke, Hjortdal, \& Kvien, 2003).

Relations between health behaviors and GSE were low. In addition, coefficients remained nonsignificant for two out of six analyses conducted for health behaviors (smoking and vigorous physical activity). This result is in line with assumptions of SCT (Bandura, 1997). The more behavior-specific the cognitions are, the stronger the relation with a target behavior is expected to be. Meta-analyses on relations between domain-specific self-efficacy and health behaviors or quality of life in cancer patients usually show that those relations are moderate in size (cf. Graves, 2003; Floyd, Prentice-Dunn, \& Rogers, 2000; Yarcheski, Mahon, Yarcheski, \& Cannella, 2004).

Because of its generality, GSE was weakly related to specific behaviors. This relation, however, might be observed for a variety of behaviors, not only one target behavior (for further evidence, see Luszczynska, Gibbons, et al., 2004; Piko, Luszczynska, Gibbons, \& Tekozel, in press).

Across samples, GSE was related to adaptive, problem-focused coping with stress. Previous studies on GSE have been focused on its relations to passive or emotion-oriented coping. Negative associations between GSE and distraction strategies or emotion-focused strategies were also found in people with chronic and acute diseases (Endler, Kocovsky, \& Macrodimitris, 2001). Among chronic pain patients, passive coping with pain was related to lower GSE (Brown \& Nicassio, 1987). The results of the present study support the assumption that GSE is related to active coping, namely, more frequent employment of planning, fighting spirit, usually seen as a predictor of successful adaptation to stressful encounters.

The four meta-analyses have achieved the most successful synthesis of the data at this time. In sum, the four population effect sizes $(.25, .28, .14$, and .28), based on diverse samples, provide the currently best-known associations between GSE and the corresponding sets of health-related constructs.

The results of the present study show that relations between GSE and other variables were of similar magnitude, regardless of country or sample. Besides the intercultural and intracultural diversity that might result from differences in education and socioeconomic status, more similarities than discrepancies were found. The study results support SCT, suggesting that a cross-cultural commonality of beliefs about efficacy and relations between these beliefs and other constructs might be expected (Bandura, 2002). These assumptions, however, have not yet been tested across countries that differ in social, economical, and cultural background. Preliminary conclusions might be drawn that similar patterns of relations exist between GSE and social-cognitive constructs, well-being, health behaviors, and coping with stress across the samples and countries (Scholz et al., 2002).

Given the circumstances of the data collection, the present study has various limitations. The samples we used in this study were probably not representative 
of the corresponding countries. Participants within and across countries differed in terms of proportions of gender and age. The majority of the constructs were assessed only in some samples, not across countries. In addition to the role of culture and language, the direct effects of other variables (such as social and economic status) that might moderate the associations between self-efficacy and related constructs should be considered.

All our hypothesized relations between GSE and other variables were confirmed by the data. The size of the coefficients was low or moderate, except for the relation between GSE and self-regulation. GSE is connected to a broad range of psychological constructs pertaining to various domains of human functioning, and it may be a useful addition to situation-specific self-efficacy measures in future studies within and across cultures. The strength of the GSE construct lies in its generality. Although the relations between GSE and some other constructs are rather weak, they are considerable for a variety of domains of human functioning.

\section{REFERENCES}

Aaronson, N. K., Ahmedzai, S., Bergman, B., Bullinger, M., Cull, A., Duez, N. J., et al. (1993). The European Organization for Research and Treatment of Cancer QLQ-C30: A quality-of-life instrument for use in international clinical trials in oncology. Journal of the National Cancer Institute, 85, 365-376.

Ainsworth, B. E., Sternfeld, B., Richardson, M. T., \& Jackson, K. (2000). Evaluation of the Kaiser physical activity survey in women. Medicine and Science in Sports and Exercise, 32, 1327-1338.

Ashby, J. S., \& Kottman, T. (2000). The relationship between personality priorities and affect, depression, self-efficacy, and fear of intimacy. Journal of Individual Psychology, 56, 172-183.

Bandura, A. (1997). Self-efficacy: The exercise of control. New York: Freeman.

Bandura, A. (2002). Social cognitive theory in cultural context. Journal of Applied Psychology: An International Review, 51, 269-290.

Bonetti, D., Johnston, M., Rodriguez-Marin, J., Pastor, M., Martin-Aragon, M., Doherty, E., \& Sheehan, K. (2001). Dimensions of perceived control: A factor analysis of three measures and an examination of their relation to activity level and mood in a student and cross-cultural patient sample. Psychology and Health, 16, 655-674.

Brekke, M., Hjortdal, P., \& Kvien, T. K. (2003). Changes in self-efficacy and health status over 5 years: A longitudinal observational study of 306 patients with rheumatoid arthritis. Arthritis and Rheumatism, 49, 342-348.

Brislin, R. W. (1970). Back-translation for cross-cultural research. Journal of Cross-Cultural Psychology, 1, 185-216.

Brown, G. K., \& Nicassio, P. R. (1987). Development of questionnaire for the assessment of active and passive coping strategies in chronic pain patients. Pain, 31, 53-64.

Carver, C. S. (1997). You want to measure coping but your protocol's too long: Consider the Brief COPE. International Journal of Behavioral Medicine, 4, 92-100.

Cervone, D. (1997). Social-cognitive mechanisms and personality coherence: Selfknowledge, and situational beliefs. Psychological Science, 8, 43-50.

Contento, I. R., Randell, J. S., \& Basch, C. E. (2002). Review and analysis of education measures used in nutrition education intervention research. Journal of Nutrition Education and Behavior, 34, 2-25. 
Endler, N. S., Kocovsky, N. L., \& Macrodimitris, S. D. (2001). Coping, efficacy, and perceived control in acute versus chronic illnesses. Personality and Individual Differences, 30, 617-625.

Floyd, D. L., Prentice-Dunn, S., \& Rogers, R. W. (2000). A meta-analysis of research on protection motivation theory. Journal of Applied Social Psychology, 30, 407-429.

Fuchs, R. (1997). Psychologie und körperliche Bewegung. Grundlagen für Theoriegeleitete Interventionen [Psychology and physical activity. Foundations for theory-based interventions]. Göttingen, Germany: Hogrefe.

Gollwitzer, P. M. (1999). Implementation intentions: Strong effects of simple plans. American Psychologist, 54, 493-503.

Graves, K. D. (2003). Social-cognitive theory and cancer patients' quality of life: A metaanalysis of psychosocial intervention components. Health Psychology, 22, 210-219.

Hampton, N. Z. (2000). Self-efficacy and quality of life in people with spinal cord injuries in China. Rehabilitation Counselling Bulletin, 42, 66-74.

Hautzinger, M. (1988). Die CES-D Skala. Ein Depressionsmeßinstrument für Untersuchungen in der Allgemeinbevölkerung [The CES-D Scale: A self-report depression scale for research in the general population]. Diagnostica, 34, 167-173.

Hunter, J. E., Schmidt, F. L., \& Jackson, G. B. (1982). Meta-analysis. Cumulating research findings across studies. Beverly Hills, CA: Sage.

Leganger, A., Kraft, P., \& Røysamb, E. (2000). Perceived self-efficacy in health behavior research: Conceptualisation, measurement and correlates. Psychology and Health, 15, 51-69.

Luszczynska, A. (in press). Wstepna adaptacja polskiej wersji narzedzia do oceny leku przed bólem Fear of Pain Questionnaire-III. [The preliminary adaptation of a Polish form of Fear of Pain Questionnaire-III]. Studia Psychologiczne.

Luszczynska, A., Diehl, M., Gutiérrez-Doña, B., Kuusinen, P., \& Schwarzer, R. (2004). Measuring one component of dispositional self-regulation: Attention control in goal pursuit. Personality and Individual Differences, 37, 555-566.

Luszczynska, A., Gibbons, F. X., Piko, B., \& Tekozel, M. (2004). Self-regulatory cognitions, social comparison, perceived peers' behaviors as predictors of nutrition and physical activity: A comparison among adolescents in Hungary, Poland, Turkey, and USA. Psychology and Health, 19, 577-593.

Luszczynska, A., \& Schwarzer, R. (2003). Planning and self-efficacy in the adoption and maintenance of breast self-examination: A longitudinal study on self-regulatory cognitions. Psychology and Health, 18, 93-108.

McNeil, D. W., \& Rainwater, A. J. (1998). Development of the Fear of Pain Questionnaire-III. Journal of Behavioral Medicine, 21, 389-411.

Petosa, R. L., Suminski, R., \& Hortz, B. (2003). Predicting vigorous physical activity using social cognitive theory. American Journal of Health Behavior, 27, 301-310.

Piko, B. F., Luszczynska, A., Gibbons, F. X., \& Tekozel, M. (in press). A culture-based study of personal and social influences of adolescent smoking. The European Journal of Public Health.

Radloff, L. S. (1977). The CES-D scale: A self-report depression scale for research in the general population. Applied Psychological Measurement, 3, 385-401.

Renner, B., \& Schwarzer, R. (2003). Risk and health behaviors. Documentation of the scales of the research project: "Risk Appraisal Consequences in Korea" (RACK). Berlin: Freie Universität Berlin.

Rimm, H., \& Jerusalem, M. (1999). Adaptation and validation of Estonian version of the General Self-Efficacy Scale. Anxiety, Stress, and Coping, 12, 329-345.

Rodgers, W. M., Hall, C. R., Blanchard, C. M., McAuley, E., \& Munroe, K. J. (2002). Task and scheduling self-efficacy as predictors of exercise behaviour. Psychology and 
Health, 27, 405-416.

Scholz, U., Gutiérrez-Doña, B., Sud, S., \& Schwarzer, R. (2002). Is general self-efficacy a universal construct? Psychometric findings from 25 countries. European Journal of Psychological Assessment, 18, 242-251.

Schulz, U., \& Mohamed, N. E. (2004). Turning the tide: Benefit finding after cancer surgery. Social Science and Medicine, 59, 653-662.

Schwarzer, R. (1991). Meta-analysis software. Version 5.3. Dubuque, IA: Brown.

Schwarzer, R., \& Jerusalem, M. (1995). Generalized Self-Efficacy Scale. In J. Weinman, S. Wright, \& M. Johnston (Eds.), Measures in health psychology: A user's portfolio. Causal and control beliefs (pp. 35-37). Windsor, England: NFER-NELSON.

Schwarzer, R., Mueller, J., \& Greenglass, E. (1999). Assessment of perceived general selfefficacy on the Internet: Data collection in cyberspace. Anxiety, Stress, and Coping, 12, $145-161$.

Schwarzer, R., \& Renner, B. (2000). Social-cognitive predictors of health behavior: Action self-efficacy and coping self-efficacy. Health Psychology, 19, 487-495.

Sherer, M., Maddux, J. E., Mercandante, B., Prentice-Dunn, S., Jacobs, B., \& Rogers, R. W. (1982). The Self-Efficacy Scale: Construction and validation. Psychological Reports, 51, 663-671.

Sniehotta, F. F., Scholz, U., \& Schwarzer, R. (in press). Bridging the intention-behaviour gap: Planning, self-efficacy, and action control in the adoption and maintenance of physical exercise. Psychology and Health.

Tursky, B., Jamner, L. D., \& Friedman, R. (1982). "The pain perception profile." A psychophysical approach to the assessment of pain report. Behavior Therapy, 13, 171-183.

Yarcheski, A., Mahon, N. E., Yarcheski, T. J., \& Cannella, B. L. (2004). A meta-analysis of predictors of positive health practices. Journal of Nursing Scholarship, 36, 102-108. 